\title{
Laser Processing of Quartz for Microfluidic Device Fabrication
}

\author{
A. Ben Azourt ${ }^{2}$, M. Vazquezt $^{1}$, B. Paullt ${ }^{1}$, and D. Brabazon ${ }^{2}$ \\ Irish Separation Science Cluster, National Centre for Sensor Research, Dublin City University, Ireland \\ ${ }^{2}$ National Centre for Plasma Science and Technology, Dublin City University, Ireland
}

\begin{abstract}
This paper presents a fast fabrication process of microfluidic channels with quartz substrates. Microchannels were ablated on the surface of quartz samples with a $\mathrm{CO}_{2}$ laser. Double sided Pressure Sensitive Adhesive (PSA) was applied to bond the samples with scribed microchannels to flat glass sheets. Dimensions of the fabricated channels were characterised with optical microscopy and laser profilometry. The recorded data was modelled with a Box-Behnken experiment design using Response Surface Methodology. Characterisation included also the measurement of optical transmission through the processed glass and measurement of flow rate through the fabricated channels. With an average width of $165 \mathrm{pm}$ and depth of 280pm, fabricated channels had appropriate dimensions for a range of microfluidic applications. A significant width of the laser processed channels provided $100 \%$ transmission for a wide range of the optical spectrum. These fabricated channels were also shown to not significantly retard the fluid flow rate thus making these channels applicable for integration into numerous detection systems for chemical separation applications.
\end{abstract}

Keywords: Laser processing, CO2 laser, microfluidic, DoE, quartz, separation science

\section{Introduction}

Microfluidics devices are widely used in research and in chemical, environmental and biotechnology sensing applications. They are fabricated to handle small quantities of fluids, allow for higher sensitivity in detection, and their function may incorporate several laboratory functions in a single chip [1]. Glass is one of the preferred materials for fabrication of microfluidic chips due to its chemical neutrality and physical stability. Typically, micro-fabrication on glass surfaces is performed using lithography and $\mathrm{HF}$ etching techniques [2]. Laser processing is an attractive cost-effect alternative offering faster and more flexible design. In last few years, many groups focused on implementation of femtosecond lasers for glass processing [3]. In most of these works, the glass surface is processed followed by various bonding techniques to form the 3D micro-feature $[4,5]$. However more recently, researchers were able to create sub-surface channels within glass materials [6]. While the results of these researches showed the possibility of microfabrication of $3 \mathrm{D}$ features with glass materials, the expensive prices of femtosecond lasers remain a major obstacle to transfer these techniques to industrial applications. Glass materials absorb strongly at or near 10 p.m making the $\mathrm{CO}_{2}$ laser very efficient for 
machining these materials [7, 8]. Previous comparison of the $\mathrm{CO}_{2}$ laser processing of four different types of glass has shown that quartz-based glass material presented more uniform channel topologies and better transmission capabilities [9,10]. However, the transmission capabilities of these channels were reduced, due to the deposition and adhesion of carbon residue during the laser ablation process. In this work, based on these previous results, a $\mathrm{CO} 2$ laser operated in pulsed mode was used to analyse the effect of laser processing parameters on the geometry of the fabricated channels' features. Characterisation of the transmission capabilities and flow rates through the formed channels were performed to assess the applicability of these microfluidic systems for separation applications.

\section{Experimental Setup}

Topology Characterisation. Fused quartz samples of $20 \mathrm{~mm}$ by $20 \mathrm{~mm}$ and $2 \mathrm{~mm}$ thick (Multi-lab Ltd, UK) were used in this study. Channels of $15 \mathrm{~mm}$ length were fabricated on the surface of the samples using a $1.5 \mathrm{~kW} \mathrm{CO} 2$ laser in pulsed mode (Rofin DC015). The laser beam with a $90 \mathrm{Rm}$ spot size was focused co-incident with an air or argon jet at one bar perpendicular to the sample surface for channel processing. The sample was mounted on a translation stage which allowed linear movement at selectable speed settings. The results of previous design of experiment models were used to set the range of the laser parameters [9]. Following the laser processing, the samples were cleaned and rinsed in deionised water in an ultrasonic bath for five minutes. From transverse sections of the samples, width and depth of the channels, were measured using an optical microscope (Reichart ME F2) and image analysis software (Beuhler Omnimet Enterprise). Width was also confirmed with a laser profilometer [11].

Design of Experiments. The experiments were designed based on a three level BoxBehnken design with full replication (central point was repeated five times) [12]. Laser power, $\mathrm{P}$, pulse repetition frequency, PRF, and sample scanning speed, $\mathrm{U}$, were set as the independent laser processing input variables, see Table 1. Response Surface Methodology was applied to the experimental data using statistical software, Design-Expert V8, for statistical analysis. A step-wise regression method was used to fit second order polynomials to the data and to identify the relevant model terms [13]. A total of 17 experiments were carried out according to the software design in a random order to avoid any systematic error.

Table 1 Three level experimental input variables settings used for power, pulse repetition freauencv and sample scanning speed.

\begin{tabular}{|l|l|l|l|}
\hline Variables & $\mathbf{- 1}$ & $\mathbf{0}$ & $\mathbf{+ 1}$ \\
\hline P [W] & 6 & 8 & 10 \\
\hline PRF [Hz] & 160 & 194 & 228 \\
\hline $\mathbf{U}[\mathbf{m m} / \mathbf{m i n}]$ & 300 & 400 & 500 \\
\hline
\end{tabular}


Optical Transmission Measurement. Optical transmission properties of fabricated channel were measured by illuminating channels with light from a tungsten halogen calibrated light source (Ocean Optics LS-1-CAL) via $50 \mathrm{tm}$ optical fibre. Transmitted light was collected by a $100 \mathrm{tm}$ optical fibre and measured using Ocean Optics MayaPRO 2000 spectrometer set at an integration time of $100 \mathrm{~ms}$. In order to evaluate the impact of the shape of the channel on light diffraction, the collecting fibre attached to a micro-stage with a resolution of $2011 \mathrm{~m}$ was moved along the width of the channel. Transmitted spectrum were recorded every $4011 \mathrm{~m}$ over a $2.5 \mathrm{~mm}$ distance, centred within the middle of the channel, see Fig. 1. Previous experiments showed that the triangular shape of the channel would diffract the light. This technique permitted the assessment of the extent of the diffraction on the transmission capabilities of the fabricated channels. Initially, the transmission intensity of non-processed quartz samples was recorded as a reference spectrum.

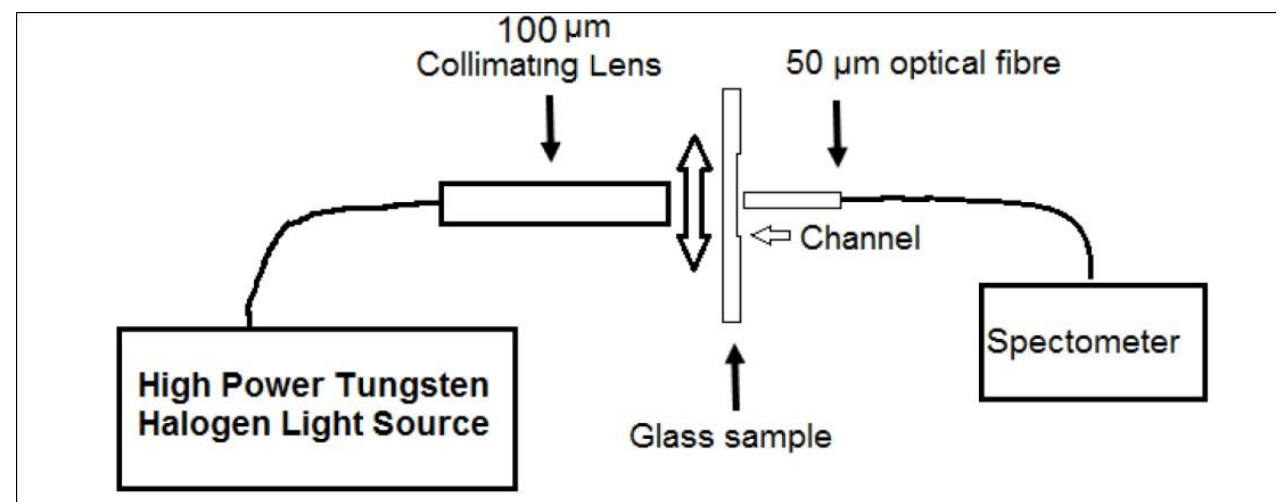

Figure 1 Schematic of the experimental set-up for the spectral transmission measurements through the fabricated channels.

Flow Rate Measurement. Using Pressure Sensitive double sided Adhesive, 6011m thick, (PSA, 3M), a compressive bonding technique was applied to bond the laser-ablated glass to flat glass sheets and thereby create the 3D microfluidic structures. The flat glass sheet was drilled using a diamond drill (Diamadental, UK) in order to create inlets and outlets for the fabricated system.

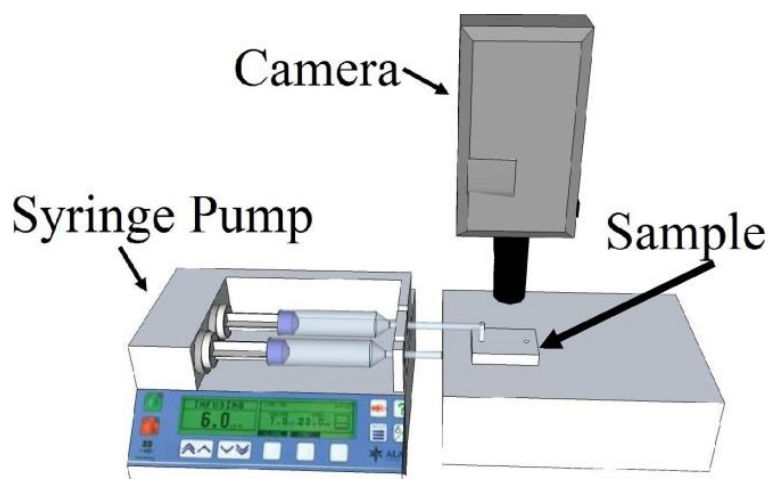

Figure 2 Schematic of experimental set-up for fluid flow rate measurements. 
For flow rate measurement, polyamide seeding particles, $511 \mathrm{~m}$ size (Dantec Dynamics, Denmark), were suspended in deionised water by sonication for 30 minutes. This suspension fluid was injected in the channel using a syringe pump at various flow rates. The particle flow was then observed with a high speed camera (Citius C10, Finland) for recording fluid flow rate, see Fig 2.

Recorded measurement speeds were compared to theoretical values. The average flow velocity $\mathrm{V}$ was calculated as:

$$
V=\frac{Q}{h w}
$$

where $\mathrm{Q}$ is the volumetric flow rate (volume/time) as set on the syringe pump control; $\mathrm{h}$ and $\mathrm{w}$ are the height and width of the flow channel's cross-section respectively.

The velocity distribution is expressed as a two dimensional power function as follows [14 16]:

$$
U=2.12 \bar{U}\left[1-\left(\frac{y}{a}\right)^{2.2}\right]\left[1-\left(\frac{z}{a}\right)^{2.2}\right]
$$

where $U$ is the average velocity in the channel; $a$ is half of depth of channel; $y$ is the width of the channel at the position of the particle; and $\mathrm{z}$ is the depth position of the particle as measured from the centre of the channel. When $\mathrm{z}=0$, the particle is in the centre of the channel.

\section{Results}

Channel Topology Results. Table 2 shows the average width and depth measurements of all fabricated samples. The sequential F-test for significance of the regression model and the individual model terms, and the lack of fit test were performed. Choosing a modified step-wise regression method with two factors interaction (2FI) modelling method, for experiments with compressed air, lead to eliminating the insignificant model terms automatically [12]. For the recorded results from the experimental work with argon, a second order quadratic model was found to best fit the data.

The adequacy measures R2, adjusted R2 and predicted R2 for the two models were all close to 1 and in reasonable agreement indicating an adequate model. The adequacy precision was greater than 4 indicating adequate model discrimination [17]. From the experimental results run with argon and after response surface analysis, equations (3) and (4) were generated as the process model. Equations (5) and (6) were generated to model the relation between the laser process run with air and the resulting channel geometries. 
Table 2 Channel width and depth dimensions after laser processing with argon and air.

\begin{tabular}{|l|c|c|c|c|}
\hline & \multicolumn{2}{|c|}{ With argon } & \multicolumn{2}{c|}{ With compressed air } \\
\hline & Width ham] & Depth ham] & Width ham] & Depth ham] \\
\hline 1 & 174 & 216 & 143 & 275 \\
\hline 2 & 162 & 238 & 218 & 441 \\
\hline 3 & 146 & 197 & 145 & 246 \\
\hline 4 & 182 & 251 & 157 & 403 \\
\hline 5 & 158 & 242 & 130 & 247 \\
\hline 6 & 178 & 284 & 225 & 460 \\
\hline 7 & 125 & 185 & 153 & 136 \\
\hline 8 & 150 & 243 & 174 & 410 \\
\hline 9 & 164 & 241 & 206 & 397 \\
\hline 10 & 170 & 255 & 153 & 342 \\
\hline 11 & 144 & 219 & 184 & 345 \\
\hline 12 & 146 & 228 & 168 & 207 \\
\hline 13 & 170 & 240 & 169 & 348 \\
\hline 14 & 162 & 241 & 175 & 319 \\
\hline 15 & 165 & 249 & 159 & 345 \\
\hline 16 & 166 & 239 & 160 & 342 \\
\hline 17 & 155 & 234 & 164 & 362 \\
\hline
\end{tabular}

$$
\text { Width }=291.88-29.92 x \text { Power }-1.41 x \text { PRF }+0.69 x \mathrm{U}+0.17 \mathrm{x} \text { Powerx PRF }
$$

$$
-1.0310^{-3} \mathrm{x} \mathrm{U}^{2} \text {. }
$$

Depth $=19.32+12.02 x$ Power $+2.04 \times$ PRF $-0.18 \times U+0.12 \times$ Power $x$ PRF

$$
-1.49 \mathrm{x} \text { Power }^{2}-7.5310^{-3} \mathrm{PRF}^{2} \text {. }
$$

Width $=-267.47+94.62 x$ Power $+0.29 x$ PRF $+0.16 x \mathrm{U}-0.23 \times$ Powerx PRF

$$
-0.09 x \text { Powerx U+ 2.7210 }{ }^{-3} \text { x Ux PRF. }
$$

$$
\text { Depth }=529.32+20.13 x \text { Power }-0.96 x \text { PRF }-1.04 x \mathrm{U}+0.08 x \text { Power } x \mathrm{U} \text {. }
$$

Optical Transmission Results. The tungsten halogen light source delivered a spectrum in the range of $350 \mathrm{~nm}$ to $1100 \mathrm{~nm}$. Fig. 3 shows the results of spectral transmission of the substrate in the range of 350 to $1100 \mathrm{~nm}$ for a micro-channel fabricated under argon. This figure shows the spectrum transmission relative to that recorded for transmission through the non-processed quartz.

Flow Rate Measurement. Using the syringe pump, the flow rates of the fluid at the inlet of the channels were varied. Table 3 summarises the results of these experiments as well as the theoretically calculated speeds for similar settings. The recoded values of velocity of the neutrally buoyant polyamide particles were taken as an average value from three measurements. For each of these measurements, a particle judged to be in the middle of the channel was tracked. Corresponding distance and time was noted for calculation of each particle speed. 


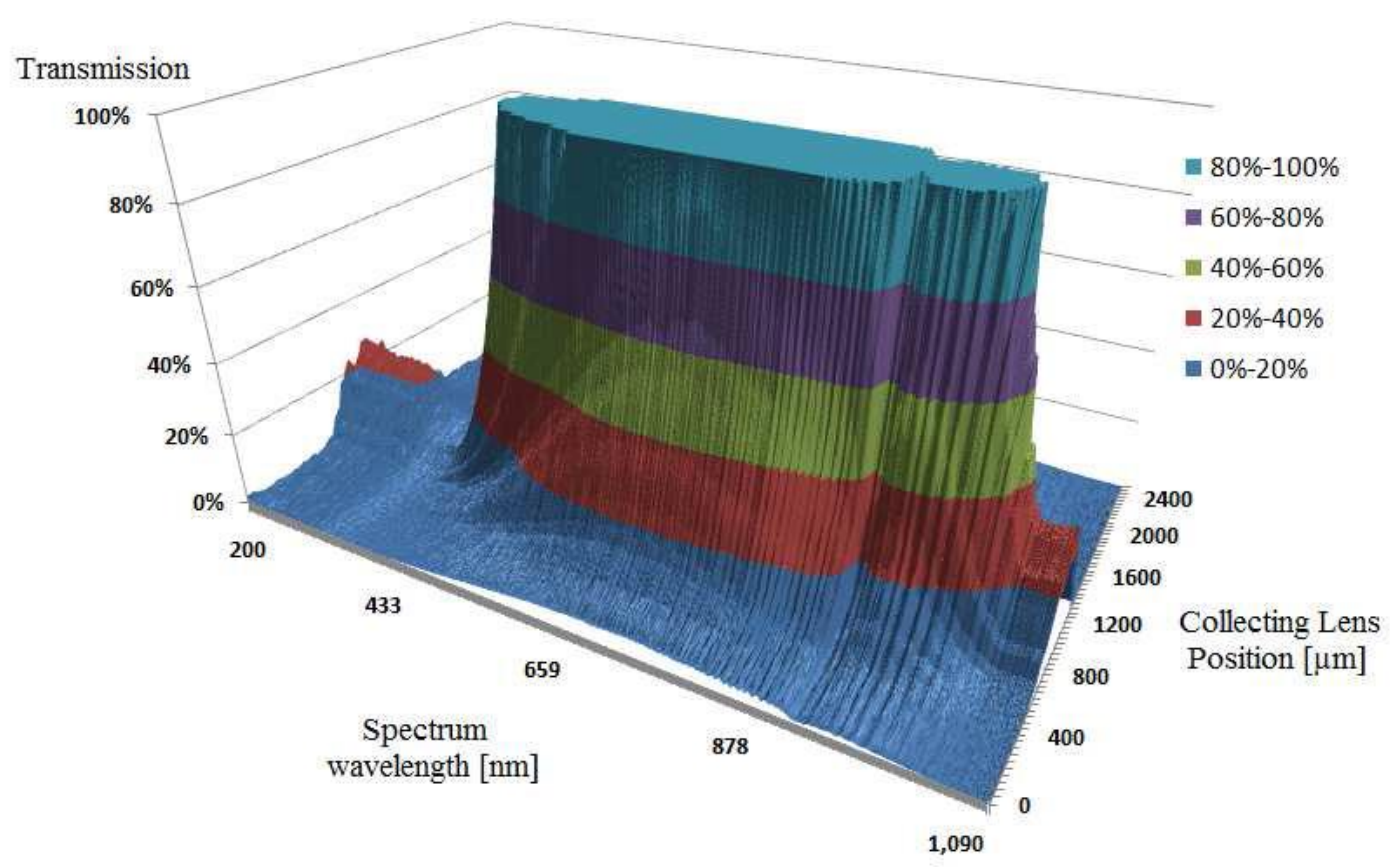

Figure 3 Optical transmission profile, relative to transmission through non-treated flat quartz sheets, for laser processed quartz micro-channels under argon atmosphere.

Table 3 Theoretical and experimental flow rate measurement results for the three set flow rates.

\begin{tabular}{|c|c|l|}
\hline $\begin{array}{c}\text { Flow rate of syringe pump } \\
{[\mathbf{m} / \mathbf{h}]}\end{array}$ & $\begin{array}{c}\text { Particle Velocity } \\
{[\mathbf{m m} / \mathbf{s}]}\end{array}$ & \multicolumn{1}{|c|}{$\begin{array}{c}\text { Theoretical Velocity } \\
{[\mathbf{m m} / \mathbf{s}]}\end{array}$} \\
\hline 0.1 & 7.67 & 8.45 \\
\hline 0.3 & 26.74 & 29.07 \\
\hline 0.5 & 37.82 & 48.66 \\
\hline
\end{tabular}

\section{Discussion}

Channel Topology. Figures 4 (a) shows the actual response versus predicted response for the channel width for set of experiments with argon. The relation between the actual and predicted responses is close to a $45^{\circ}$ line on this equiaxed plot. Therefore, the model can be seen to adequately describe the response within the limits of the factors investigated in this study. Figures 4 (b) shows the interaction effect among the parameters within the investigated range for channel depth for set of experiments with compressed air. It can be seen that the power, $\mathrm{P}$, had the strongest effect. Changing $\mathrm{P}$ from the minimum to maximum values increased the responses by about $40 \%$. The speed, $\mathrm{U}$, also had a strong impact on the depth of the channels as it controlled the time of exposure of the surface to the beam. Changing sample speed from the minimum to maximum values decreased the width and depth responses. Increases in Pulse Repetition Frequency, on the other hand, produced a reduction in channel width but a negligible effect on channel depth. 


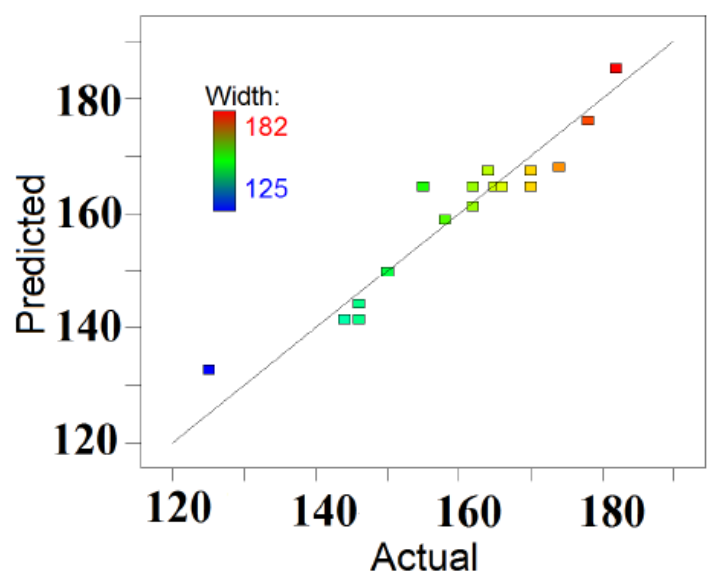

(a)

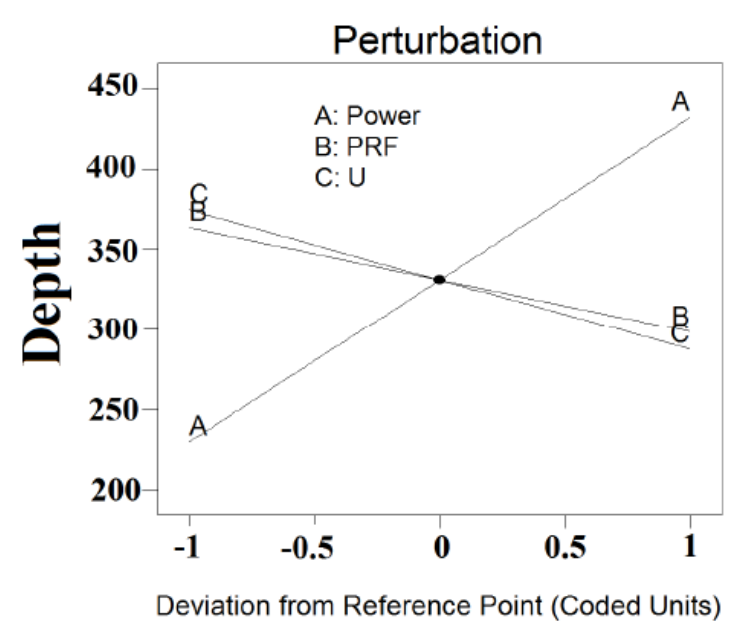

(b)

Figure 4 (a) Model predicted versus actual width measurements (for processing in argon) and (b) model interaction effects of the DoE parameters for depth ( for samples processed in air)

Overall, P had the strongest effect on the channel size, width and depth. Sample scanning speed, U, also had a considerable effect on depth of the channels. Recorded measurements showed no major difference on channel geometries between channels fabricated with argon or compressed air. Samples fabricated under argon had slightly smaller dimensions which could be attributed to the reduced percentage of oxygen during the processing with argon inhibiting sample surface burning and associated rise in temperature.

Optical Transmission Measurement. The application of argon during laser processing improved the transmission capability of the fabricated channels which is believed to be due to less surface burning. As shown in Fig. 3, fabricated channels presented $100 \%$ transmission of spectrum from $480-1050 \mathrm{~nm}$ within a region $60011 \mathrm{~m}$ wide. However, the transmission of the lower wavelengths $(350-500 \mathrm{~nm})$ did not exceed $60 \%$. This can be explained by the high angle of diffraction of light at this wavelength with quartz which has an index of refraction of 1.46. These results demonstrate the feasibility of using these samples for various chromatography detection techniques for separation applications.

Flow Rate Measurement. Initial experiments have shown that the fabricated channel presented a smooth surface that did not adversely affect the flow rate of fluids injected inside these fabricated microfluidic channels. From these results, it can be confirmed that the channels have already the potential to be applied for bio-chemical separation applications.

\section{Conclusion}

The developed mathematical models fitted well to the experimental data within the investigated range of parameters allowing widths and depths of processed channels to be predicted. The light transmission measurements showed that the application of argon gas improved the transmission capabilities of fabricated micro-channels. Future work will focus on further assessing the fluid flow capabilities of fabricated channels for fluids of different constitutive behaviour and for separation applications. 


\section{Acknowledgements}

This work was carried out with the financial support of Science Foundation Ireland under Grant No. 8/SRC/B1412.

\section{References}

[1] Mark D et al, Chemical Society Review, Vol 39, No 39, pp 1153-1182, 2010

[2] Mendonca C.R. et al, Applied Surface Science ,Vol 254 pp 1135-1139, 2007

[3] Gattass, R. and Mazur, E., Nature Photonics, Vol 2, pp 219 - 225, April 2008

[4] Queste, S. et al, Microsyst Technol, Vol 16. pp 1485-1493, February 2010

[5] Schafer DN et al, CLEO/QELS 2009

[6] Yang L. et al, Optics Letters, Vol 35. Issue 19, pp 3225-3227, Oct 2010

[7] Duley W.W., Laser processing and analysis of materials, Plenum Press, 1982.

[8] Bansal N.P and Doremus R.H., Handbook of glass properties, Academic Press Inc., 1986

[9] Ben Azouz, A. et al, Europhysics Conference Abstracts, Vol. 34A. (2010)

[10] Ben Azouz, A et al, Nanotech '10 Conference, Anaheim, USA, pp.496-499 (2010)

[11] Issa A. et al, AMPT2006, Las Vegas, USA, NI16, 103, pp. 1 - 5, August 2006

[12] Montgomery D.C., Design and analysis of experiments, John Wiley \& Sons Inc. (2008)

[13] K. Miuraet et al, Applied Physics Letters., Vol. 71, pp. 3329-3331 (1997)

[14] Reynolds 0., Philosophical Transactions of the Royal Society of London,pp 935-982 (1883)

[15] Information B. et al, Advanced Drug Delivery Reviews, 55(3), pp. 349-377 (2003)

[16] Wang, H., \& Wang, Y. Measurement, 42(1), 119-126. (2009).

[17] Design-Expert software, "v8, user's guide, Technical manual", Stat-Ease Inc. $(2010$ 